\title{
Vital Symptoms Monitoring and Classification of Bipolar Disorder
}

\author{
M.Kiruthiga Devi, R.Kaviya
}

\begin{abstract}
Bipolar disorder is a mental illness that puts patients into extreme states of mind known as mania and depression. These mental states are very harmful to the lives of the patients as their day to day actions are disrupted. This project aims at identifying the symptoms of the patients who have extreme moods to determine if they are bipolar using sensors and smart phones. Patients with mental illness tend to exhibit symptoms like reduced physical activity, changes in mood, drastic changes in sleep pattern, inability to cope with stress and withdrawn from socializing. These changes can be monitored using sensors and the data collected is compared with the data collected from healthy individuals. A classification algorithm is applied to the data collected to classify the symptoms and detect if the person has bipolar disorder or is just showing subtle signs of mood swings.
\end{abstract}

Key Words: Bipolar disorder, Random forest classification, Neural network, DDMLP, Machine learning.

\section{I.INTRODUCTION}

Bipolar disorder is a type of mental illness that puts people into a cycle of mood swings. It has two cycles namely mania and depression. Mania is a state when the patient exhibits a lot of energy through increased body movement, social behaviour and lack of sleep and restlessness. Depression is the counter of mania during which the patient is sad most of the time, does not move from bed, spends his time doing nothing and has suicidal thoughts. Bipolar disorder is rare among children as they are not prone to it. But it is a very common mental disorder in the case of adults. This disorder can be caused due to several reasons, some of them being hereditary. Also traumatic life situations can be the major cause of this disorder. This disorder can only be diagnosed and not cured completely. But it can be kept under check and guidance if diagnosed early on time.

There are several mental illnesses that are prevalent and most of the illness have similar symptoms. It requires doctors to monitor the patient personally by conducting reviews and counselling over a long period of time. Time is a very important aspect when it comes to mental illness as the type of disorder has to be diagnosed before the patient is deeply affected by it.
There are several symptoms exhibited by patients who suffer from mental disorders and it is important to take into account all the important symptoms. These include the amount of physical activity exhibited by the patient, his social activity and behaviour which includes number of phone calls made or attended, his social media activity etc. Also the sleeping pattern of a person is of important concern. One important diagnosis test for a person with a mood disorder is the questionnaire test which consists of a series of questions which the person has to answer. This is very vital in detecting bipolar disorder.

This project aims to ease the problem of monitoring the patient and helps in accurate diagnosis of the type of disorder. It aids in collecting real time information from the patient over a period of time and analysing it using machine learning algorithms to detect the disorder. It helps to save time spent on travel and monitoring in person and also gives precise diagnosis of the illness.

\section{RELATED WORK}

[1].This paper helps to classify the patients based on particular symptoms.Analyse the classification algorithms in order to specify the patients having unipolar or bipolar disorder.

[2].Understanding of the human and social behaviour of the patients based on smartphones.Gathering of information through sensors and it monitored by the smartphones.

[3].This paper introduces the Tshirts, by observing the variation of heart beats during the bipolar states and also acquire the heart rate variability(HRV) features.

[4].Learning of datas collected from the smartphones by collecting the number of incoming,outgoing and the attended calls of the particular patient.

[5].Usage of sensor information,such as speech and the movement activity to classify the mood of the patient.In addition to that, self reported questionnaires are gathered to set up the states.

[6].This paper introduces the concept of boosting and bagging of classification trees.Random forest algorithm helps to making priorities of decision trees and identify the results.

[7].This paper explains the various ways in which the activity of a person can be monitored including sensors, smartphones, applications etc.

[8].The questionnaire which is very vital in detecting bipolar disorder and it's stage is presented and a better understanding of the answers is provided.

[9].The random forest classification algorithm has been explained and it's pros and cons have been discussed in this paper.

[10].This paper explains the IFTTT app that is being used to collect the data and send to storage space and used for

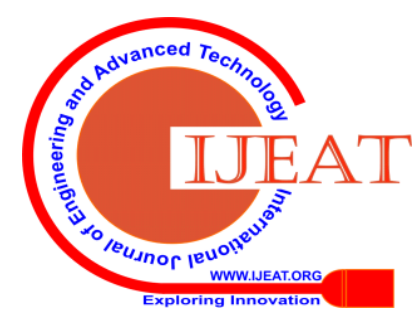


further purposes.

\section{III.EXISTING SYSTEM}

In the existing system, only a single symptom or parameter of the person has been taken into consideration. While the body movement of a person is an important symptom to be considered while diagnosing their mental illness, using just that symptom will not give the most accurate result. It is necessary to combine several parameters to detect the precise kind of mental disorder exhibited.

The existing system makes complete use of sensors like accelerometer to monitor the body movement of a person.

The previous systems also implement a different classification algorithm called as the KNN classification. This is among the oldest and easiest types of classification, but it is not very effective. This utilises a large number of different datasets and classification is hard to implement. The results are not efficient or accurate and a varied algorithm is needed to improve the performance.

\section{PROPOSED SYSTEM}

The proposed system mainly concentrates on monitoring the patient who suffer from the mood changes related troubles and classifying the collected data from the patient to detect the issue. The bipolar disorder can be identified by noting the social behaviour of the person, measuring their body movements and asking them some questions related to depression and other day to day activities of the person. For this paper, we have developed an android application using which the body movements of the patient can be recorded and answers of questionnaire can be obtained. Social behaviour monitoring focuses on the call $\log$ of patient. All three parameters dataset is stored in a database and sent to neural network for training. The datasets are classified using the random forest classifier algorithm. Once the training is over testing and validation can be done by giving inputs to the trained network.

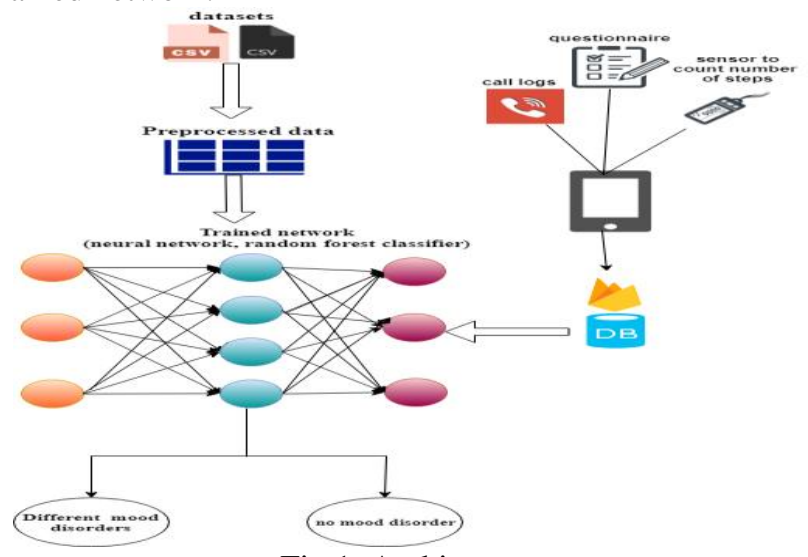

Fig 1. Architecture

\section{A. Sensors}

Sensor used to observe the body movements of a patient is pedometer. The main idea of using pedometer is to discover the type of bipolar disorder that is whether the person is in depression state or mania. If a person is in deep depression then they hardly move from one place to another. Oppositely if a person is in mania then they will be hyperactive. So counting the number of steps helps to distinguish the types. Pedometer will count the number of steps taken by a person.
The steps taken are counted by continuously observing the motion of the person using inbuilt accelerometer sensors in smartphone. The accelerometer sensor works by keenly observing the position in which device is held, the direction of the device, the angle with which it works, the speed at which it is moved and the gravity. The counts given by this sensor is accurate if the sensor is activated before starting the walking or other workouts and after completing the work. The accuracy may differ from one device to another. Approximately 90 to 95 percent accurateness is obtained using this sensor.

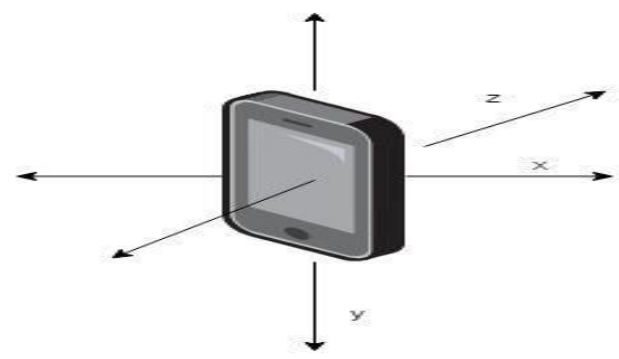

Fig 2. Accelerometer Sensor

\section{B. Social Behavior}

Social behavior focuses on monitoring the call log of the patient. If a person is depressed then the communication made by them will reduce, they like to be silent all time. In the case of mania they will be hyperactive so their interaction will be more. To monitor this, we have taken the outgoing calls and the number of calls attended by the patient. Approximately a healthy person makes 80 to 90 calls per month. If this number is increased or reduced then we have to notice that person. The number of attended calls may vary depends on the number of incoming calls a person get. Approximately a normal person may attend 3 to 6 calls per day, in the case of depressed person it is 0 to 2, 6 and above I the case of mania. These data are collected through the application and stored in the spreadsheet for further processing.

\section{Questionnaire}

A set of questions is collected from the various resources to sense the reaction of the patients. These questions are predominantly yes or no questions and they cover several aspects like the persons age, family medical history etc. These questions are asked to the patient and the responses are stored in the database. Based on the answers of the patient they will be classified.

\section{Application Design}

A mobile application has been developed to track the patient as it is the easiest way of reaching the patient and a reliable resource for the patient. Application consists of pedometer sensor which can be linked with the inbuilt accelerometer sensor of the smartphone and questionnaire sets. These values are stored in the firebase database which is an open source database and values can be converted into csv file or json file as per the user needs. The call log is examined and stored in the spreadsheets which needs further processing to count number of outgoing and attended calls. 


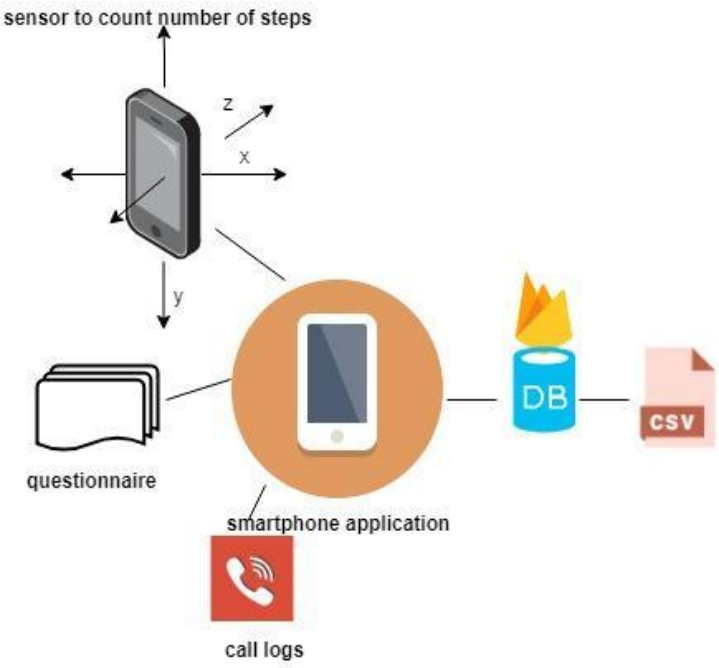

Fig 3. Application design

\section{E. Neural Network}

Neural networks are the one which establishes the exact behavior of human brain system. It has at least 3 layers. First layer is input layer which is used to get input from the user. Third layer is output layer to give the outputs. In between layer is the hidden layer which processes the input to produce output. There may be more than one hidden layers in a network. The deep neural network has many hidden layers to process the complex hidden layers. It can add more hidden layers based on the difficulty level of network. Each node in a layer is connected to every other nodes in next layer that is, a strongly connected network. We have used deep dumb multilayer perceptron (DDMLP) is one type of feed forward neural network. During the training of datasets the input is fed to input layer, the hidden layers computes the weight to next node and follow the procedure till the output layer. If the output is negative or less accurate than expected then, back propagation takes place to the initial hidden layer and again the weights computed to reach output layer through the feed forward network.

\section{F. Random Forest Classification}

One of the simplest and easiest machine learning algorithm for classification is random forest algorithm. This creates a collection of decision trees and combined which is trained using bagging method to get stable and accurate output. If the number of inputs are given to the network for training then the trained samples are taken randomly and the test set is made fit into that sample to get correct output. This avoids the underfitting issue. This main advantage is it will not suffer from overfitting. Because the trees are splitted, trained and fit into the samples randomly. The splitting of nodes will be based on the random best features from the subset of nodes. This will make the increased randomness while training the datasets and fitting them within the output. We have used 8:2 ratio for splitting the inputs to training and testing.

\section{RESULT}

\begin{tabular}{|c|c|c|c|c|}
\hline \multirow[t]{2}{*}{ Emp Id } & \multicolumn{3}{|c|}{ Parameters (Symptoms) } & \multirow[t]{2}{*}{ Disorder } \\
\hline & $\begin{array}{c}\text { Body } \\
\text { movement }\end{array}$ & Quiz & $\begin{array}{c}\text { Social } \\
\text { Behaviour }\end{array}$ & \\
\hline Emp01 & 4.82 & 1 & 90 & $\begin{array}{l}\text { Anxiety } \\
\text { Disorder }\end{array}$ \\
\hline Emp02 & 5.32 & 0 & 68 & $\begin{array}{l}\text { Bipolar } \\
\text { Disorder }\end{array}$ \\
\hline Emp03 & 1.90 & 1 & 72 & $\begin{array}{l}\text { Bipolar } \\
\text { Disorder }\end{array}$ \\
\hline Emp04 & 6.66 & 1 & 85 & $\begin{array}{c}\text { Mood } \\
\text { Disorder }\end{array}$ \\
\hline Emp05 & 5.87 & 1 & 81 & $\begin{array}{l}\text { Anxiety } \\
\text { Disorder }\end{array}$ \\
\hline Emp06 & 3.25 & 0 & 79 & $\begin{array}{c}\text { Mood } \\
\text { Disorder }\end{array}$ \\
\hline Emp07 & 3.90 & 0 & 53 & $\begin{array}{c}\text { Personality } \\
\text { Disorder }\end{array}$ \\
\hline Emp08 & 2.74 & 1 & 74 & $\begin{array}{l}\text { Bipolar } \\
\text { Disorder }\end{array}$ \\
\hline Emp09 & 4.92 & 0 & 91 & $\begin{array}{l}\text { Anxiety } \\
\text { Disorder }\end{array}$ \\
\hline Emp10 & 6.37 & 1 & 63 & No disorder \\
\hline
\end{tabular}

Table 1. Diagnosis of disorder based on symptoms

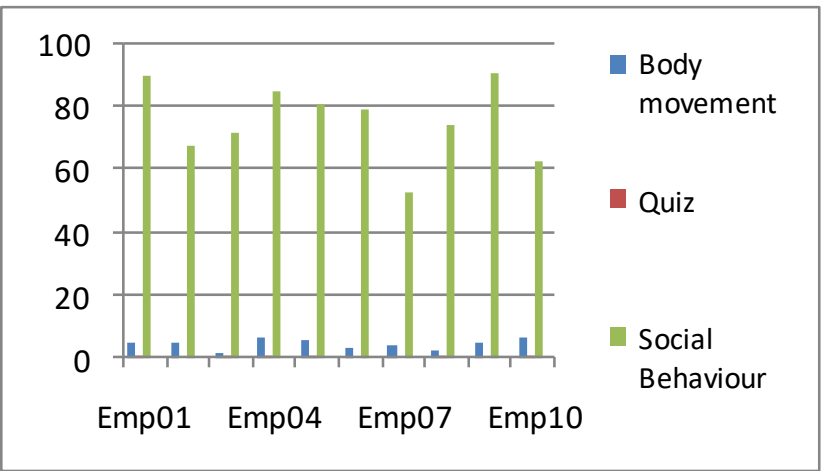

The above mentioned table, Table 1 shows the various disorders diagnosed from the given inputs. Based on all the three symptoms the mental disorders are being classified. 


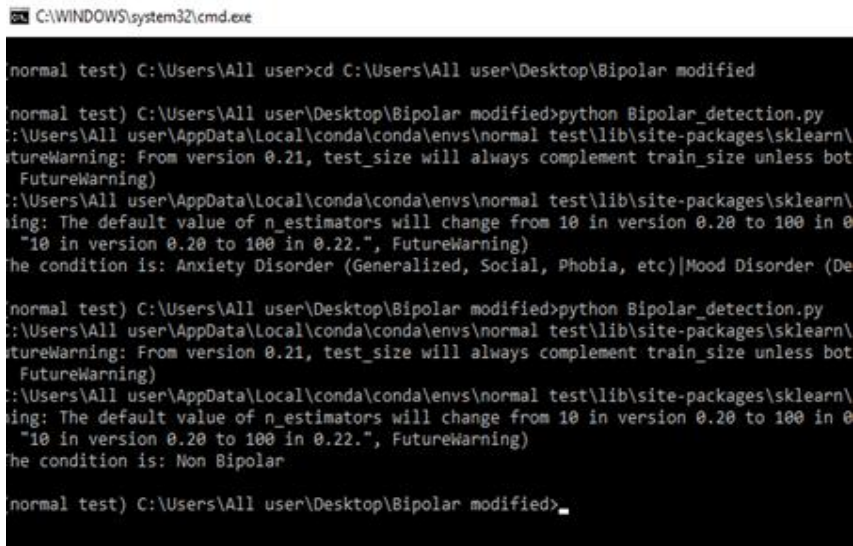

Fig 4. output 1

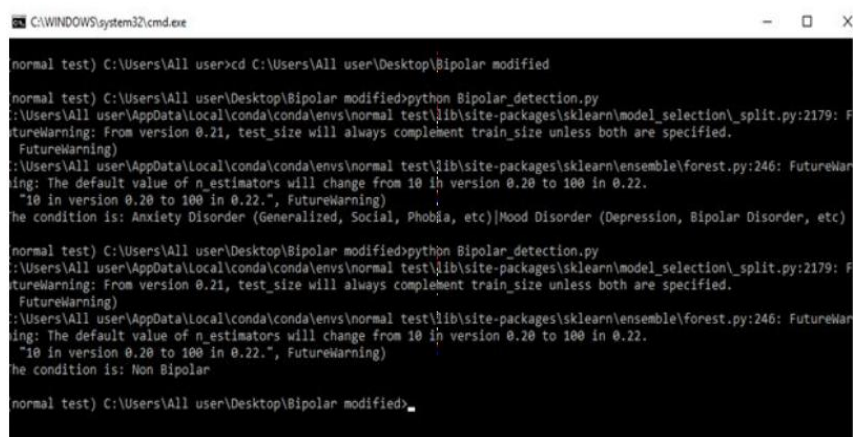

Fig 5. output 2

The above mentioned figures 4 and 5 display the output which is the disease that is diagnosed based on the symptoms. The values entered in table 1 are given to the random forest classification algorithm which then classifies each patient based on their symptoms. These classified disorders are shown as in fig 4 and 5 respectively.

\section{CONCLUSION}

We have used the random forest classification to classify the patient because in terms of medical related application it gives more accuracy than any other algorithms. We have got almost 90 to 95 percent accuracy using this algorithm. This is implemented in real time and tested for some patients. The training is done for previously available patient datasets and tested for the new patients.

\section{FUTURE SCOPE}

The real time medical applications take more data than we use. In case of the larger datasets and storage the accuracy of random forest algorithm reduces. Over fitting occurs when the number of inputs increased in this algorithm. This will not affect training but, testing totally confuses. In turn wrong predication is given as output. To avoid this we can use the rainforest algorithm for larger datasets if it's implemented in real time medical applications. And more parameters can be added to improve the accuracy.

\section{REFERENCES}

1. Enrique Garcia Ceja, Michael Alexander Riegler,PetterJackobsen, T.Nordgreen "Motor activity based classification of depression in unipolar and bipolar patients" IEEExplore International Conference,July 2018 .doi:10.1109/CBMS.2018.00062
2. Wouter B. Teeuw, Johan Koolwaaij, Arjan Peddemors "Monitoring human behaviour through mobile technology",October 2018,MDPI journel,doi:10.3390/proceedings2191243

3. Sara Mariani, Matteo Migliorini, Giulia Tacchino, Claudio Gentili "Clinical state assessment in bipolar patients by means of HRV features obtained with a sensorized T-shirt ", August 2018,doi 10.1109/EMBC.2012.6346408

4. Maria Faurholt-jepsen,maj Vinberg,mads Frost,sune Debel,ellen Margrethe Christensen,jakob E Bardram "Behavioral activities collected through smartphones and the association with illness activity in bipolar disorder",April 2016. doi: 10.1002/mpr.1502.

5. A. Maxhuni, A. Munoz-Mel endez, V. Osmani, H. Perez, O. Mayora, and E. F. Morales "Classification of bipolar disorder episodes based on analysis of voice and motor activity of patients",Volume 31 Issue C, September 2016.doi:10.1016/j.pmcj.2016.01.008.

6. A Liaw, M Weiner "Classification and regression by random forest", R news, Vol. 2/3, December 2002.

7. C. Burton, B. McKinstry, A.S.Tatar, A. Serrano-Blanco, C. Pagliari, and M. Wolters, "Activity monitoring in patients with depression: a systematic review," Journal of affective disorders, vol. 145, no. 1, 2013.

8. "Development and Validation of a Screening Instrument for Bipolar Spectrum Disorder: The Mood Disorder Questionnaire” Robert M.A Hirschfeld , M.D., Janet B.W. Williams, The American Journal of Psychiatry, Nov 2000.

9. "Random Forest: A Classification and Regression Tool for Compound Classification and QSAR Modeling" Vladimir Svetnik, Andy Liaw, Journal of chemical information and modelling, Nov 2003.

10. "Automate the Internet With If This Then That (IFTTT)", Steven Ovadia, Behavioural and Social Sciences Librarian, 2014. K Vijayakumar and C. Arun, "A Survey on Assessment of Risks in Cloud Migration", International Journal of Applied Engineering Research, ISSN 0973-4562 Vol. 10 No.66 May 2015.

11. K. Vijayakumar and C. Arun, "Continuous Security Assessment of Applications in Cloud Environment", International Journal of Control Theory and Applications, ISSN: 0974-5645 volume No. 9(36), Sep 2016, Page No. 533-541.

12. R.Joseph Manoj, M.D.Anto Praveena, K.Vijayakumar, "An ACO-ANN based feature selection algorithm for big data", Cluster Computing The Journal of Networks, Software Tools and Applications, ISSN: 1386-7857 (Print), 1573-7543 (Online) DOI: 10.1007/s10586-018-2550-z, 2018.

\section{AUTHORS PROFILE}

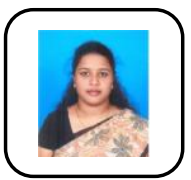

M.Kiruthiga Devi is working as an Assistant professor/CSE in Sri Sairam engineering college, Chennai. she had published papers in reputed journals and conferences. she is having 7 years of experience in teaching. Her areas of interest are data science,Machine Learning, Deep Learning ,Artificial intelligent ,WSN,Human Computer Interaction and. She also member in IAENG.

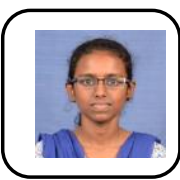

Kaviya $\mathbf{R}$ is a Computer Science and engineering graduate from Sri Sairam Engineering College. She has previously published a paper on Internet of Things internationally. Her interests are majorly in the field of data science and artificial intelligence. She has also done several projects on IOT and AI. Her papers can be found on the IEEE Xplore library and also in scopus index.

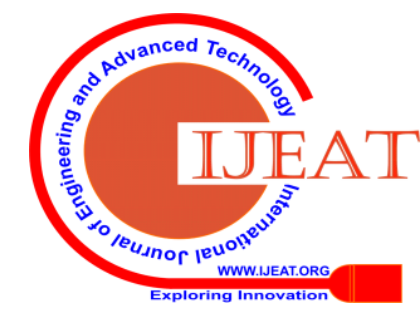

